A CASE OF

LATERAL SINUS THROMBOSIS WITH PYÆMIC PULMONARY AND PERICARDIAL COMPLICATIONS, ETC.; OPERATION ; RECOVERY.

BY JOHN MACKIE, L.R.C.P. EDIN., $\Lambda N D$

H. BELL TAWSE, M.B. ABERD., F.R.C.S. ENG.

NoTwITHSTANDING the almost weekly reports of recovery after lateral sinus thrombosis we consider the following case sufficiently uncommon to be worthy of addition to the list.

The patient, a well nourished boy, aged 15 years, had constant discharge from the left ear for eight years, with attacks of earache when the discharge lessened. The pain abated after poulticing, and free discharge of foul pus relieved the other symptoms. For the past two or three years these attacks were accompanied by mastoid pain, which in recent months was more acute. Lately more severe symptoms had been superadded during an attack and giddiness, distressing tinnitus, sickness and vomiting, with acute general headache, restlessness, and delirium developed, before a copious discharge of pus-a discharge so profuse that seemingly it could not have come from the tympanum alone-relieved his condition. On Sept. 26th, 1906, a more severe attack than usual led his parents to call in Dr. Harvey Francis of Arnold, who recoonising the serious nature of the case telephoned for Mr. Mackie. The boy was found to be exceedingly ill with all the symptoms of serious extension of his ear trouble. The left meatus was full of foul granulations, and appliances for the removal of these being at hand it was decided to clear these out and to await developments for a few hours. At this time there were pain and tenderness over the mastoid and the temperature was $104^{\circ} \mathrm{F}$., but there was no evidence of a thickened jugular vein and the cervical glands were not enlarged. On the next day there was no improvement. If anything the patient seemed worse. His temperature had ranged from $105^{\circ}$ to $97^{\circ}$, accompanied by rigors and sweating, leading to a diagnosis of lateral sinus thrombosis. Immediate operation was decided upon. The mastoid process was exposed by the ordinary incision behind the ear, and on chiselling through the cortex the anatomy was found in rather a confused state. The mastoid antrum was continuous with the cavity of a seemingly chronic extradural abscess, the tegmen antri and tympani being replaced by soft, pulpy, foul-smelling granulation tissue. On removal of this the dura mater was exposed over a large area, and working backwards towards the sinus through dirty washleather-looking material the sinus was reached, the vein wall being unrecognisable as such. The thrombosis was not disintegrated and came away quite easily, free bleeding being established from both ends. No pus was found in the sinus. On commencing the operation the question of tying the vein was left an open one, and at this stage, taking into account the collapsed condition of the patient and the free bleeding from both ends of the sinus, it was decided to leave the vein alone. As the disease had practically performed the radical mastoid operation the wound was partially closed and provision was made for free drainage. For the next two days the patiert was naturally in a critical condition. He had daily rigors, followed by rapid rise of temperature. On one occasion the temperature fell from $104^{\circ}$ to $96.2^{\circ}$ in a few hours. Nevertheless, the patient expressed himself as relieved, took nourishment freely and retained it, and had an average pulse of 80 beats to the minute. On the third day after operation (Sept. 30th) the temperature fell to subnormal and remained there a few hours and the patient seemed to be doing very well, but towards the evening of that day his breathing became laboured and on the next day left basal pneumonia and pericarditis were added to his troubles. Little could now be done but watch the case. The patient was full of pluck and, apparently unaware of his critical condition, was making a good fight. He remained in much the same condition till oct. 6th-nine days after the operation and five days after pneumonia was recognisedwhen the temperature, after running up to $106 \cdot 2^{\circ}$, in three hours dropped to $95^{\circ}$-a fall of $11 \cdot 2^{\circ}$. There were almost daily rigors but the pulse remained fairly strong. The appetite was good and the patient frequently expressed himself as being "all right." After this, and contrary to all expectations, matters improved and on Oct. 9th the temperature had been normal for 48 hours. On the 10th it suddenly rose to $104 \cdot 2 \circ$, a severe rigor was experienced, and the temperature fell to $99 \cdot 4 \circ$, and soon after about half a pint of thick foul-smelling blood-stained greenish pus was expectorated, evidently from a pulmonary abscess. The patient's general condition remained fairly satisfactory, with occasional rises of temperature to $101^{\circ}$ until the 13 th, when he complained of sore throat and pain about the angle of the jaw on the left side and along the course of the internal jugular vein. A swelling soon appeared in the neighbourhood of the angle of the jaw and it gradually increased until the 19th, when the neck was laid open from the mastoid tip to the sterno-clavicular articulation. The internal jugular vein, which was small and feebly pulsating, was tied at its junction with the subclavian. The ligature of the vein was temporarily followed by unilateral facial cyanosis which rather alarmed the anæsthetist. A large abscess was evacuated at the upper part of the neck, a drainage-tube was inserted, and a gauze drain was introduced down to the site of ligature. Stitches were put into the rest of the wound and were left untied until four days later. The wound healed by first intention, the abscess cavity gradually filled up and closed, the temperature remained normal, and the patient was discharged on Oct. 31st with a healthy granulating wound over the mastoid. This soon healed up and now he is in excellent health. The cervical scar became painful and markedly keloidal, but the application of $\mathrm{x}$ rays has materially reduced the thickening and dispelled the pain.

It seems very probable that in this case there was an extradural abscess of some standing, which had at intervals filled up and discharged through the meatus and that ultimately the sinus wall became involved and a thrombosis resulted. This, perhaps, is not an unusual occurrence, but to have recovery after such complications as pulmonary abscess and pericarditis is certainly very rare.

The case showed a few interesting points, such as the fall of $11.2 \circ$ of temperature in three hours, the unilateral cyanosis after ligature of the jugular vein, and the remarkable physical and mental stamina of the patient, who never realised the seriousness of his condition. Thickening along the course of the internal jugular vein, reported in most text books as being present in sinus thrombosis, was absent in this case - in fact, neither of us has seen it in previous cases.

Nottingham.

\section{A SIMPLE METHOD OF OBTAINING A PREPARATION OF LIVING ISOLATED LEUCOCYTES.}

By CONSTANT W. PONDER, M.A., B.C. CANTAB., M.R.C.S. ENG., L.R.C.P. LoND.

(From the Cambridge Pathological Laboratories.)

THE following method of preparing a field of living leucocytes, free from the other elements of the blood, was evolved during the course of a piece of research work, and it seemed to me that it might be useful to those working with leucocytes and could also be used to demonstrate to the stadent in a striking way the living entity of the white blood cell.

No unusual apparatus is necessary. A warm stage, made by a strip of copper, one end of which is heated, while the other, resting on the microscope, has a hole corresponding to that in the stage; a white porcelain staining dish for washing the slide; and a material which can be obtained anywhere, "plasticine," or modelling clay, which is used to prepare the blood-chamber hereinafter described, are all that is required. An incubator at blood temperature is a nseful refinement. A bath of warm, normal, saline solution $(0.75$ per cent.) at blood temperature is to be prepared in the porcelain dish, and can be kept ready by resting it on the hotter end of the copper strip. A point can easily be found where its temperature will not vary more than a degree or two from $38^{\circ} \mathrm{C}$. This simple arrangement is shown in the diagram (Fig. 1). 
A blood chamber is prepared as follows. A morsel of plasticine much smaller than a pea is rolled ont with a glass slide until it is about one and a half inches long and as thick as the lead of a pencil, and then bent round in a spiral way and fixed in the middle of another clean glass slide, as shown in Fig. 2, forming a chamber $A$, with an exit E. A fairly

FIG. 1.

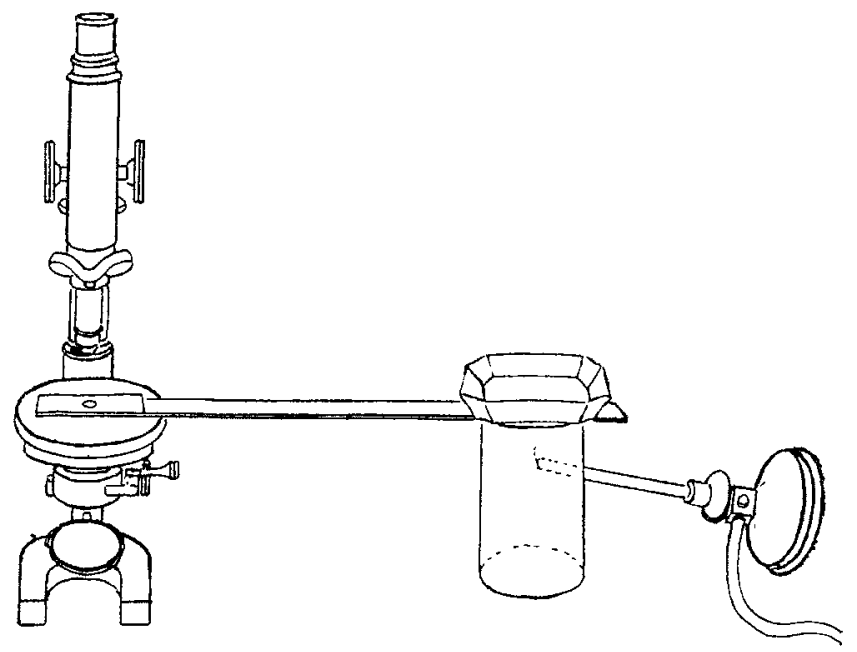

A general view of the apparatus.

large drop of fresh blood is obtained and allowed to fall into the chamber at $A$; a cover-slip is superimposed and pressed down carefully with another slide, so that as the clay flattens blood and air are driven out through the exit $\mathrm{E}$, which must always remain patent, and a thin, even layer of blood fills the chamber and the exit passage; this is shown in Fig. 3.

FIG. 2.

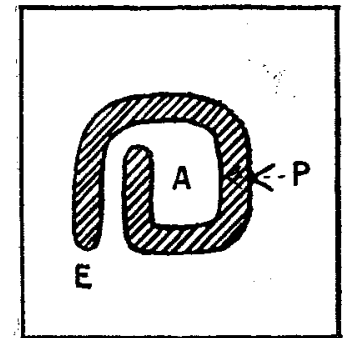

A, Chamber occupied by B, Blood. P, Ring of plasticine. E, Exit.

A little practice is necessary to perform this successfully, but unless it is wished to keep the leucocytes alive several hours, an air bubble in the chamber does no harm. The slide is now placed in the incubator at blood temperature, or simply in the bath of warm saline solution which is at a temperature of about $38^{\circ} \mathrm{C}$, and it is left here 15 minutes. During this period the blood clots and the leucocytes escape, apparently by their own activity, and passing both upwards and downwards seek the glass surface of the slide and the cover-slip in hundreds, to which they adhere tenaciously. In order to get rid of the clot and serum the cover-slip is carefully removed and both cover-slip and slide are placed in the warm saline bath, which has been prepared ready; the remains of the clot and plasticine are removed with fine forceps or needles and both cover-slip and slide are washed gently by passing them to and fro under the saline solution; this process is continued until the free red corpuscles are all washed away, as seen by the disappearance of the reddish colour. If now the cover-slip or slide be held up to the light an even grey film can be seen with the naked eye, which consists entirely of leucocytes, adhering to the surface of the glass. Two preparations can now be made; one, by placing a clean cover-slip over the film on the slide, moistened with saline solution; the other by placing the cover-slip on which is a film of leucocytes on a clean slide, also allowing a little saline solution to interpose ; in the former case the leucocytes are lying on the upper surface of the slide, in the latter case they are hanging from the lower surface of the cover-siip, and indeed a hanging drop preparation can be made from the cover-slip. On examining with a microscope either of these preparations the field is seen to be crowded with active leucocytes of every shape, and the appearance is not masked nor the movements hindered by the presence of red blood cells or fibrin.

If it is desired to bring in contact with these leucocytes any fluid, such as one containing a suspension of bacteria, and watch the result, it can be done by the hanging drop method, or better by placing the cover-slip on which is the film of leucocytes face downwards on a plasticine chamber similar to the one originally described, but which contains a drop of the fluid, such as a suspension of bacteria, and pressing it down to form another chamber in the way described for the original blood. In this way a clear view of what takes place is obtained and evaporation cannot occur to any detrimental degree if the exit is quite narrow. The leucocytes can be watched in conditions more approximating those of their natural habitat if the second chamber is made with a drop of serum obtained by centrifugalising the same blood as that from which they were originally obtained.

By this method living isolated leucocytes can be segregated directly from human blood and kept under observation in vitro, without further precautions, for about an hour.

Cambridge.

\section{Clinital atotes:}

\section{MEDICAL, SURGICAL, OBSTETRICAL, AND THERAPEUTICAL}

\section{A CASE OF PURE MYXOMA OF THE LOWER JAW.}

By D. W. Daniels, M.B., B.S. Lond., M.R.C.S. ENG., HOUSE SURGEON TO ST. MARY'S HOSPTTAL, PADDINGTON, $W$.

THE patient, a woman, aged 27 years, first noticed a hard lump in the outer side of the gum of the lower jaw nine months ago. It gave her no pain. The tumour slowly grew and began to get painful. In October last it started to grow more rapidly.

On admission there was a swelling of about the size of a marble opposite the bicuspid teeth of the left lower jaw. The tumour was firm and hard all over. There was no fluctuation. No obvious swelling could be seen on the cheek.

An incision was made over the swelling between the gum and cheek. It was then found that the tumour readily shelled out entire, leaving a cavity in the bone, extending right through to its inner margin.

Microscopical examination showed the tumour to be a pure mxyoma, unmixed with any other tissue, sarcomatous or otherwise.

I have to thank Mr. A. J. Pepper for permission to publish these notes.

St. Mary's Hospital. W.

\section{RUPTURE OF THE LIGAMENTUM PATELLÆ.}

\section{By Dr. Paravicixi.}

SEEING from an annotation in your pages ${ }^{1}$ and from a letter which appeared the following week that cases of rupture of the ligamentum patellæ have been seldom recorded, I venture to contribute the following notes. The case offers the special feature that no direct violence to the tendon itself took place, the hyperflexion of the knee and simultaneous contraction of the quadriceps having been the only possible causes.

The accident happened to myself some six years ago in the Swiss mountains. Running down a steep slope covered with a thin layer of snow I slipped and fell, my left gluteal region touching the left heel, my left leg being completely doubled. I felt a cracking and an acute pain and found myself unable to stretch the left leg by its own force. Through my trousers I noticed a gap below the patella which was drawn up by the quadriceps extensor. There was no external wound nor the slightest sign of a contusion. I was alone and only able to crawl slowly. After having called for help during two hours I 1 The Lancet, Scpt. 12th, p. 825. 